\title{
Autopercepção da condição de saúde bucal em idosos institucionalizados e não institucionalizados
}

\author{
Self-perception of oral health condition \\ of the institutionalized and non institutionalized elders
}

Evanildo Henrique M acêdo da Costa ${ }^{1}$

Maria Vieira de Lima Saintrain ${ }^{1}$

Anya Pimentel Gomes Fernandes Vieira ${ }^{1}$

Abstract This study evaluated the self-perception of oral health condition of institutionalized and non institutionalized elder population using a oral examination and a questionnaire divided in three parts: demographic data, general health and the GOHAI index. A total of 96 individuals above 60 years old living in Fortaleza, Brazil, were interviewed. They were divided in two groups, G1 - institutionalized ( $n=48)$ and $G 2$ - noninstitutionalized $(n=48)$. The mean age of the study population was 69,8 years (SD 7,5) and $70,8 \%$ of the interviewed were woman. $62 \%$ had some type of medical treatment in the last year, and despite the fact that the majority $(84.4 \%)$ of the elders thought that their general health was good or regular, the average number of teeth presented was 3.9 in both groups. Among them, 47.9\% were using superior prosthesis and $26 \%$ inferior prosthesis. The GOHAI average was 17.53 qualifying asnegativetheself-perception of oral health. There was no difference between the two groups regarding physical and psychological aspects. Key words Oral health, Gohai index, Institutionalized and non institutionalized elder
Resumo Este estudo avaliou a autopercepção das condições de saúde bucal em idosos institucionalizados ou não, por intermédio da aplicação de um exame bucal e um questionário composto por três partes: dados demográficos, saúde geral e o índice GOH Al. A amostra foi de 96 indivíduos com 60 anos ou mais, residentes em Fortaleza, CE, Brasil, dividida em G1 - idosos institucionalizados $(n=48)$ e $G 2$ - idosos não institucionalizados $(n=48)$. A pesar de $84,4 \%$ dos idosos acreditarem que sua saúde está excelente ou razoável, a média dedentes presentesfoi de 3,9 nos dois grupos, constatando-seuma população predominantementede edêntulos. Dentreeles, 47,9\% usavam prótesetotal superior e $26 \%$ inferior. A média do GOH AI foi de 17,53 , qualificando como negativa a saúde bucal, e não houve diferença entre os dois grupos em relação aos aspectos físicos e psicol ógicos.

Palavras-chave Saúdebucal, ÍndiceGO H AI, I dosos institucionalizados e não institucionalizados
1 Universidade de Fortaleza. Av. Washington Soares 1.321, Bairro Édson Queiroz. 60.165-220 Fortaleza CE.

evanildohenrique@hotmail.com 


\section{Introdução}

A preocupação com a qualidade de vida (QV) na vel hice ganhou relevância nos últimos trinta anos, a partir do momento em que o crescimento do número de idosos e a expansão da longevidade passaram a ser experiências compartilhadas por um número crescente de indivíduos vivendo em sociedades diferentes ${ }^{1}$. A institucionalização da população idosa cresce proporcionalmente à ascensão da expectativa de vida, que ultimamente tem aumentado significativamentea necessidade deatendimento edecuidados ${ }^{2}$, que, infelizmente, nem sempre são realizados de maneira eficiente, deixando muitos asilados da terceira idade sem um acompanhamento adequado e consequentemente com situação de saúde mais precária que os não abrigados.

A Organização Mundial da Saúde (OM S) define QV como a percepção do indivíduo sobre sua posi ção na vida, no contexto da cultura e dos sistemas de valores nos quais el e vive, e em relação a seus objetivos, expectativas, padrões e preocupações ${ }^{3}$. Assim, vários fatores têm o potencial de influenciar a QV deuma pessoa, incluindo a saúde bucal dela.

$\mathrm{Na}$ perspectiva de Werner et al. ${ }^{4}$, uma boa saúde bucal deveincluir ausência de dores orofaciais, mastigação adequada, facilidade de ingestão e digestão dos alimentos. Ela também deve contribuir para a comunicação, sobretudo nos atos de falar e sorrir, que têm o potencial de aumentar a autoestima das pessoas e reduzir o número de doenças.

Enquanto o cirurgião-dentista avalia a condição clínica pela presença ou ausência de doenças, para o paciente são importantes os sintomas, os problemas funcionais e sociais decorrentes da saúde bucal. A pesar de a autopercepção não substituir o exame clínico do paciente, ela permitequese tenha um panorama mais próximo da real condição do indivíduo ${ }^{5}$.

Consoante pensem M arinelli e Sreebny ${ }^{6}$, as preocupações dos idosos com relação à saúde bucal se apresentam em décimo quarto lugar numa lista de valores de vinte queixas mais comuns nessa faixa etária. Portanto, se faz cada vez mais necessário o emprego de instrumentos para quea sociedade tenha conhecimento da condição epidemiológica da saúde bucal da pessoa idosa e possa contribuir, dessa forma, para o desenvolvimento das ações sociais de prevenção, diagnóstico eintervenção tanto para a população institucionalizada quanto para a não institucionalizada.

Dentre esses instrumentos, citam-se: perfil do impacto da saúde bucal ${ }^{7}$, levantamento da saúde bucal na qualidade de vida ${ }^{8}$, perfil do impacto das enfermidades em saúde bucal ${ }^{9}$, indicadores subjetivos do estado de saúde bucal ${ }^{10}$, medida de qualidade de vida relacionada à saúde bucal ${ }^{11}$, impactos odontológicos na vida diária ${ }^{12}$, índice geriátrico para avaliação de saúde bucal (Geriatric Oral Health Assessment Index - GOHAI) ${ }^{13}$ sendo, pois, um índice extremamente adequado para avaliar essa população.

Esteestudo teve como objetivo avaliar ecomparar a autopercepção da saúde bucal de idosos institucionalizados e não institucionalizados, buscando analisar a percepção dos pacientes em relação à saúde bucal, dados imprescindíveis para melhor orientar os profissionais nas ações de saúde e elaboração de políticas públicas como programas educativos, preventivos e curativos.

\section{Metodologia}

Idosos com mais de 60 anos foram pesquisados em uma instituição de longa permanência (Lar Torres de M elo) e um grupo de apoio a idosos (Projeto Cabelos Brancos). Existem 240 residentes no Lar Torres de M elo (G1), instituição situada no bairro Jacarecanga (Fortaleza, CE, Brasil), e 71 participantes no Projeto Cabelos Brancos do Dendê (G2), com sede no Núcleo de Assistência M édica Integrada (NAMI), situado no bairro Edson Queiroz, também em Fortaleza. Assim, a população de idosos pesquisada neste estudo pode ser dividida em dois grandes grupos: institucionalizados e não institucionalizados. 0 primeiro é constituído por aqueles que vivem em instituições delonga permanência (instituição de auxílio ao idoso), seja por motivos de desprezo por parte da família, seja por vontade própria de possuir uma vida mais tranquila, longe de preocupações diárias e de sentimentos de incômodo aos seus cuidadores. 0 segundo grupo, constituído por idosos não institucionalizados, representa os que vivem com seus cônjuges, filhos, familiares, sozinhos e/ou outros, e que participam de grupo de apoio/convivência na comunidade.

0 estudo foi desenvolvido por meio de um exame bucal simplificado e uma entrevista baseada em um questionário estruturado, que apresentava perguntas sobre dados demográficos, saúde geral do idoso eo índice GOHAI (qualidade de vida). 0 exame simplificado da cavidade oral foi realizado sob luz natural, com o auxílio de um espelho clínico e um abaixador de língua. 0 pesquisador (EH M C) foi treinado e calibrado pela coautora M VL Saintrain e analisou o nú- 
mero de dentes presentes na cavidade oral, uso e necessidade de prótese dentária.

Perguntas sobre idade, estado civil, ocupação (passada e/ou presente) e escolaridade foram realizadas na seção de dados demográficos do questionário. $\mathrm{Na}$ seção do questionário sobre saúde geral, o idoso foi indagado sobre a autopercepção de saúde (tendo como alternativas: excelente, razoável e ruim) e a comparação desta com a de outrosidosos (alternativas: melhor, igual ou pior), assim como a comparação entrea sua saúde atual eum ano atrás (alternativas: melhor, igual ou pior). Também foi perguntado se 0 idoso teve atendimento médico e dentário nos últimos três meses, ea realização de higiene dentária.

O índice GOHAl foi utilizado para avaliar, através do autorrelato do paciente, a qualidade de vida dos idosos em relação a sua saúde bucal. Esseíndice consisteem dozequestões de múltipla escol ha sobre problemas bucais que avaliam três dimensões: física (alimentação, fala e deglutição), psicossocial (cuidado com a própria saúde bucal, insatisfação com a aparência, autoconsciência re lativa à saúde bucal e o fato de evitar contatos sociais em razão de problemas odontológicos) e dor/desconforto (considerando o uso de medicamentos para al iviar essas sensações, desde que provenientes da boca ${ }^{14,15}$. As perguntas 1, 2, 3 e 4 compreendem o domínio físico, apresentando uma pontuação mínima de 4 e máxima de 12; as perguntas 6, 7, 9, 10 e 11 compreendem o domínio psicossocial e possuem valores entre 5 e 15; por último, as questões 5, 8 e 12 compreendem 0 domínio dor/desconforto, que varia de 3 a $9^{13,16}$.

Cada pergunta no Índice GOHAI apresenta três respostas possíveis: sempre/muitas vezes; às vezes/raramente; enunca - recebendo os escores 1,2 e 3, respectivamente. A exemplo de Silva et al. ${ }^{17}$, optou-se por simplificá-las em "sempre", "às vezes" e "nunca". A soma total dos escores assinalados por entrevistado compreende 0 valor do índice para o indivíduo, que pode variar de 12 a 36. Quanto mais alto for o valor do índice, melhor é classificada a saúde bucal do indivíduo e, consequentemente, melhor sua qualidade de vida autopercebida relacionada à saúde bucal. $\mathrm{Na}$ classificação geral da pontuação, de acordo com Silva et al. ${ }^{17}$, valores entre 34 e 36 são considerados altos; de 31 a 33 moderados; e menores do que 30 são havidos como baixos.

Como a análise comparativa do índice GO$\mathrm{HAl}$ entre os gruposfoi feita empregando o teste do t-independente, a amostra foi calculada tendo por base a equação de cálculo amostral deste. Valores de alfa de $5 \%$ e beta de $90 \%$ foram utili- zados no cálculo, e uma amostra de 47 indivíduos por grupo foi encontrada ${ }^{18}$.

O critério de inclusão deste estudo considerou indivíduos com 60 anos ou mais, capazes de responder ao questionário proposto. Antes da coleta de dados, foi realizado um estudo piloto, por meio da aplicação do questionário em uma amostra de oito idosos da Clínica Integrada do curso de Odontologia da Universidade de Fortaleza (Unifor). Esteestudo tevea finalidadedeuniformizar as variáveis investigadas, padronizando e sistematizando as informações obtidas, constituindo, desta forma, um mecanismo de maior confiabilidade e precisão aos dados coletados.

0 protocolo da pesquisa cumpriu os preceitoséticos deautonomia, não mal eficência, beneficência e justiça, em investigação com seres humanos, conforme consta na Resolução n 196/96 do CNS, tendo sido aprovado pelo Comitê de Ética em Pesquisa (Coética) da Unifor sob o $n^{\circ}$ 06-239/24/08/06.

Os dados foram analisados estatisticamente por meio do Software Statistical Package for Social Science(SPSS for W indows 13.0, SPSS Inc, Chicago, IL, USA ). A análise estatística do tipo descritiva teve por base 0 ato de determinar a distribuição das variáveis, enquanto a estatística analítica serviu para comparar as duas populações, sendo estabelecido o nível de significância de $5 \%$.

\section{Resultados}

Um total de 96 indivíduos entre 60 e 94 anos, média 69,8 anos ( $D P \pm 7,5$ ), foi pesquisado durante os meses de setembro e outubro de 2006. A pesquisa foi conduzida com 48 idosos de uma instituição de longa permanência - grupo institucionalizado (G1) - e 48 idosos de um grupo de apoio a idosos - grupo não institucionalizado (G2). Houve maior participação de mulheres (70,8\%), de idosos sem escolaridade $(55,2 \%)$ e de aposentados $(73,9 \%)$ na pesquisa.

Um total de $84,4 \%$ dos entrevistados acredita quesua saúdeestá excelenteou razoável, enquanto $59,4 \%$ creem que sua saúde está melhor do que a média da saúde das pessoas do seu convívio. Já $47,9 \%$ acham que sua saúde estava meIhor no momento da pesquisa do que no ano anterior. De acordo com o teste Qui-quadrado, não houve diferença entre a autopercepção de saúde nos dois grupos analisados ( $p>0,05)$.

No que concerne ao acesso aos serviços de saúde, $62 \%$ dos entrevistados relataram ter recebido algum tipo de atendimento médico, porém 
não foi informado que tipo de atendimento foi realizado. Quanto à saúde bucal, oito $(8,3 \%)$ entrevistados referiram ter obtido tratamento odontológico nos últimos três meses; no entanto, sete destes entrevistados consideraram tratamento odontológico o fato de terem participado de exame odontológico realizado em outras pesquisas. Utilizando o teste Q ui-quadrado, notouse que não existia diferença entre acesso a tratamento odontológico nos dois grupos $(p>0,05)$.

De acordo com os dados obtidos, a maioria $(93,8 \%)$ respondeu que fazia algum tipo de higieneoral. Setenta e cinco por cento real izavam sua higiene por meio de escovação, 18,7\% com bochecho e $2,1 \%$ com fio dental.

Em relação ao número de dentes dos entre vistados, constatou-se que $58(60,4 \%)$ da população estudada era desdentada total, que a média de dentes nos pesquisados era de 3,9 eque apenas $6,2 \%$ da população possuía vinte ou mais dentes na boca - verificando-se um alto índice de extração/perda dentária nessa população em detrimento do tratamento preventivo e conservador.

Em relação à prótese, foi observado que $51 \%$ dos entrevistados a utilizavam, sendo que $47,9 \%$ usavam prótese total superior, $26 \%$ total inferior, $1 \%$ removível parcial superior e $1 \%$ removível parcial inferior. Por meio do teste do Quiquadrado, observou-se não haver significância estatística $(p=0,066)$ para maior uso de prótese entre os idosos não institucionalizados (G2) e institucionalizados (G1).

Sobre o valor total do índice GOHAI, não houve nenhuma diferença considerada significante, quando analisadas as populações de idosos institucionalizados e não institucionalizados (teste t-independente; $p>0,05$ ). É importante enfatizar o fato de que ambas as populações possuem o valor médio total do GOHAI menor do que 30 , considerado baixo como valor relacionado à saúde bucal pela autopercepção do paciente, de acordo com Silva et al. ${ }^{17}$. A penas um indivíduo do grupo de idosos institucionalizados (G1) eum do grupo não institucionalizados (G2) apresentaram o valor total do GOH Al acima de 30, o que classifica indivíduos com boa saúde bucal ${ }^{17}$. O maior valor total do GOHAl foi 34 (considerado alto) no G1 e 31 (considerado moderado) no $\mathrm{G} 2$.

Em relação ao domínios físico (alimentação, fala e deglutição), psicológico e dor/desconforto, não houve nenhuma diferença estatística, quando comparadas as duas populações ( $\mathrm{G} 1$ e G2) (Tabela 1).

\section{Discussão}

Segundo dados do último levantamento epidemiológico realizado no país, SB Brasil ${ }^{19}$, a condição de saúde bucal da população idosa apresenta-se fora das metas preconizadas pela Organização M undial da Saúde(OM S) e pela Federação Dentária Internacional (FDI) ${ }^{20}$, que diz que pelo menos 50\% dos idosos entre 65 e 79 anos devem ter pelo menos vinte dentes funcionais na cavidade bucal. No Brasil, apenas $10 \%$ desses idosos possuem mais que esse número de dentes, demonstrando o predomínio do edentulismo (com uma média de 25,8 dentes extraídos, de um total de 32 dentes) ${ }^{19}$. No presente estudo, o valor encontrado foi ainda mais baixo, com apenas seis indivíduos (6,2\%) com mais de vinte dentes.

Adicionalmente, detectou-se neste estudo a média de apenas 3,9 dentes por indivíduo pesquisado, quando a OMS preconiza um mínimo de vinte dentes para uma mastigação satisfatória $^{20}$. Esses indicadores levam à reflexão de Ro-

Tabela 1. Distribui ção e porcentagem dos valores totais do Índice GOH Al e de seus domínios nos dois locais da pesquisa - Fortaleza (CE), 2006.

\begin{tabular}{|c|c|c|c|c|c|}
\hline & \multicolumn{4}{|c|}{ GOHAI } & \multirow{2}{*}{$\begin{array}{c}\text { Teste } \\
\text { t-independente } \\
\text { entreG } 1 \text { e G2 } \\
\text { (valor de p) }\end{array}$} \\
\hline & $\begin{array}{l}\text { Possibilidades } \\
\text { de pontuação }\end{array}$ & $\begin{array}{c}\mathrm{G} 1+\mathrm{G} 2 \\
\text { Pontos (\% do valor } \\
\text { máximo possível) }\end{array}$ & $\begin{array}{c}\mathrm{G} 1(\mathrm{n}=48) \\
\text { (pontos) }\end{array}$ & $\begin{array}{c}\mathrm{G} 2(\mathrm{n}=48) \\
\text { (pontos) }\end{array}$ & \\
\hline GOHAI total & $12-36$ & $17,5313(48,69 \%)$ & 17,6250 & 17,4375 & 0,806 \\
\hline Domínio físico & $4-12$ & $5,8654(44,88 \%)$ & 5,5208 & 5,2500 & 0,406 \\
\hline Domínio psicológico & $5-15$ & $8,0104(53,40 \%)$ & 7,4500 & 8,2708 & 0,184 \\
\hline Domínio Dor/desconforto & $3-9$ & $4,1354(45,95 \%)$ & 4,3542 & 3,9167 & 0,098 \\
\hline
\end{tabular}

G1 = Lar Torres de M elo; G2 = Cabelos Brancos do Dendê. 
drigues e colaboradores ${ }^{21}$, quando estes situam a escassez de programas de saúde bucal dirigidos a idosos - segundo eles, por não representarem prioridade no serviço público, mesmo em face dos problemas acumulados e da forte mudança demográfica verificada no país. Tal afirmação corrobora os dados encontrados nesta pesquisa, em que apenas $8,3 \%$ dos entrevistados tiveram algum tipo de atendimento odontológico no último ano, considerando ainda que, deste total, 7,3\% foram referentes à pesquisa e não a tratamento preventivo ou curativo.

É importante observar que, com esse peque no número de dentes, praticamente toda a população estudada necessita de reabilitação protética. No entanto, apenas $51 \%$ dos idosos faziam uso de prótese dentária, havendo uma predominância da prótese total superior $(47,9 \%)$ sobrea inferior $(26 \%)$, condição esta justificada pelos entre vistados em razão do desconforto e da má adaptação das peças, principalmente da inferior. A capacidade mastigatória, afetada pelas extrações, pode ser em parte recuperada pelo uso de próte$\mathrm{se}^{22}$. M origuchi ${ }^{23}$ indica que a perda da dentição natural influi em diversos aspectos do organismo (estéticos, pronúncia, digestão e, principalmente, mastigação). Segundo 0 autor ${ }^{24}$, mesmo com 0 uso da prótese dentária, a capacidade mastigatória do indivíduo é reduzida para $25 \%$.

Em relação ao índice geriátrico para avaliação de saúde bucal, não houve nenhuma diferença significativa entre os valores encontrados para os idosos institucionalizados (G1) e os não institucionalizados ( $G 2)(p=0,806)$. As somas dos escores - conforme adaptado de Silva et al. ${ }^{17} \mathrm{em}$ sempre, al gumas vezes enunca - tiveram valores variando de 12 a 36. N esta classificação, valores entre 34 e 36 são considerados altos, de 31 a 33 são moderados, e menores do que 30 são havidos como baixos. 0 grupo, como um todo, apresentou um valor total do GOHAI considerado baixo, comportando uma percepção negativa das condições de saúde bucal, pois valores menores do que 30 são tidos como a pior situação em termos de qualidade de vida inerente à saúde bucal para esse índice.

É importante ressaltar que, apesar de o Índice GOHAI da população estudada haver apresentado escores inferiores a 30 (valor considerado baixo), para a maioria dos idosos - mediante a entrevista preliminar ao teste GOHAI - sua saúde foi considerada excelente ou razoável e melhor quando comparada às pessoas de seu convívio. Bulgarelli eM anço ${ }^{25}$ detectaram em sua pesquisa com idosos que $65,1 \%$ deles estavam satisfeitos e/ou muito satisfeitos com a própria saúde bucal. Em outro estudo ${ }^{26}$, os idosos reconheciam a importância dos dentes naturais; contudo, não percebiam como a perda dentária poderia alterar a saúde. Essa contradição leva a uma reflexão de que os idosos possam considerar como "natural" a má condição de saúde bucal. Em idosos, a percepção também pode ser afetada por valores pessoais, como a crença, visto que algumas dores e incapacidades são inevitáveis nessa idade, o que pode levar a pessoa a superestimar sua condição bucall ${ }^{5,16}$. Assim, de acordo com Sarti ${ }^{27}$, o sentimento de dor dos idosos é visto como um fenômeno natural, tornando mais difícil conceber para si a ideia de bem-estar. $\mathrm{Na}$ lição de Buss ${ }^{28}$, a nova concepção de saúde importa uma visão afirmativa quea identifica como bem-estar equalidade devida enão simplesmente como ausência de doença.

Pelos resultados deste estudo, pode-se concluir pela necessidade de se trabalhar cada vez mais a fim de que haja uma reformulação no serviço público odontológico em todos os grupos etários para se evitar/prevenir o endentulismo na população de idade avançada. Adicionalmente, énecessário que, para a população idosa, sejam desenvolvidas, além de ações educativas e preventivas, medidas reabilitadoras. Com efeito, é importante ressaltar que o tratamento do idoso deve ser realizado de maneira diferenciada em virtude das mudanças fisiológicas decorrentes do envel hecimento, presença de doenças sistêmicas ecrônicas ealta incidência de deficiênciasfísicase mentais. 0 idoso não é simplesmente mais um paciente esim um indivíduo que exigedo profissional um preparo prévio para poder atender às suas reais necessidades com muita paciência e dedicação29.

\section{Conclusão}

Apesar dea maioria dos idosos (84,4\%) acreditar que sua saúde geral está razoável ou excelente, a autopercepção avaliada pelo GOHAl acerca da saúde bucal foi muito negativa nos dois grupos, apresentando baixos valores, média de 17,6 para idosos institucionalizados (G1) e17,4 para os não institucionalizados(G2). Não houve diferençano valor total do Índice GOH Al entreos grupos $\mathrm{Gle}$ G2. Assim, faz-se necessário que políticas públicas sejam desenvolvidas para o melhoramento da saúde bucal da população estudada. 


\section{Colaboradores}

EH M Costa é o responsável pela pesquisa e pela metodologia referente ao trabalho de conclusão (TCC) do Curso de O dontologia da Universidade deFortaleza. M VL Saintrain eAPGF Vieira, orientadora e coorientadora, respectivamente, trabaIharam na concepção e na redação final do artigo.

\section{Agradecimentos}

À Dra. Fátima Sales, coordenadora do Projeto Cabelos Brancos do Dendê; em especial à Dra. Liana e ao Lar Torres de M elo, particularmenteà Dra. Júlia eà Sra. Alzira Souza, pela ajuda na realização das entrevistas.

\section{Referências}

1. Neri AL. Qualidade de vida na velhice e atendimento domiciliário. In: Duart, AOD. Atendimento domiciliar: um enfoque gerontológico. São Paulo: Atheneu; 2000. p. 33-47.

2. Reis SCGB, Higino MASP, Melo HMDM, Freire MCM . Condição de saúde bucal de idosos institucionalizados em Goiânia-GO, 2003. Rev Bras Epidemiol 2005; 8(1):67-73.

3. The WHOQOL Group. The World Health Organization quality of life assessement (WHOQOL): position paper from the World Health Organization. Soc Sci M ed 1995; 41:1403-1410.

4. Werner CW, Sauders MJ, Paunovich E, Yehm CK. Odontologia geriatric. Rev FOL 1998; 11(1):62-70.

5. Kiyak HA. Age and culture: influences on oral health behavior. Int Dent J 1993; 43:9-16.

6. Marinelli RD, Sreebny LM. Perception of dental needs by the well elderly. Spec Care Dent 1982; 2(4):161-164.

7. Slade GD. Development and evaluation of oral health impact profile. Community Dent $\mathrm{H}$ ealth 1994; 11(1):3-11.

8. Cornell JE. Oral health quality of life inventory. Behav M ed 1994; 4(3):257-272.

9. Reisine $S$. Oral health and the sickness impact profile. Journal Public Health 1997; 35(2):1-19.

10. Locker D. Subjective oral health status indicators. Community Health $1997 ; 27(3): 257-270$.

11. Kressin NR. The oral health-related quality of life measure. J Dent Educ 1997; 6:494-497.

12. Leão ATT, Sheiham A. The development of measures of dental impacts on daily living. Community Dental Health 1996; 13:22-26.

13. Atchison KA. The general oral health assessment index. Community Dent Oral Epidem 1990; 24(4): 385-389.

14. Atchison KA, Dolan TA. Development of the Geriatric Oral Health Assessment Index. Journal of Dental Education 1990; 54(11):680-687.

15. Pinto VG. Identificação de problemas. In: Pinto VG. Saúde bucal coletiva. 4a ed. São Paulo: 2000. p. 139-222.

16. Silva SRC, Fernandes RAC. Autopercepção das condições de saúde bucal por idosos. Rev Saude Publica 2001; 35(4):349-355.
17. Silva DD, Sousa MLR, Wada RS. Autopercepção e condição de saúde bucal em uma população de idosos. Cad Saude Publica 2005; 21(4):1251-1259.

18. Normam GR, Triner DL. Biostatistics: the bare essentials. London: BC Decker Inc; 2000.

19. M inistério da Saúde. Projeto SB Brasil 2003 - Condições de saúde bucal da população brasileira 2002-2003: resultados principais. Brasília: Coordenação N acional de Saúde Bucal; 2004.

20. Fédération Dentaire Internationale (FDI). Global goals for oral health in 2000. Int Dent J 1982; 32(1):7477.

21. Rodrigues SM, Vargas AM D, Moreira AN. Percepção de saúde bucal em idosos. Revista Arquivo em Odontologia (Faculdade de O dontologia da UFM G) 2003; 3:195-212.

22. Colussi CF, Freitas SFT. Aspectos epidemiológicos da saúde bucal do idoso no Brasil. Cad Saude Publica 2002; 18(5):1313-1320.

23. Moriguchi $Y$. Aspectos geriátricos no atendimento odontológico. Revista Odonto-Ciências 1990; 9:117123.

24. Moriguchi Y. Aspectos geriátricos no atendimento odontológico. Odontólogo M oderno 1992; 19:11-13.

25. Bulgarelli $A F, M$ anço $A R X$. Idosos vivendo na comunidade e a satisfação com a própria saúde bucal. Cien Saude Colet 2008; 13(4):1165-1174.

26. Unfer B, Braun K, Silva CP, Pereira Filho LD. Autopercepção da perda de dentes em idosos. Interface 2006; 9(18):217-226.

27. Sarti CA. A dor, o indivíduo e a cultura. Revista Saúde e Sociedade 2001; 10(1):2-8.

28. Buss PM. Promoção da saúde e qualidade de vida. Cien Saude Colet 2000; 5(1):163-177.

29. Freire RM, Santos JFF, Damião CF, M archini L. Saúde bucal dos pacientes idosos institucionalizados. Revista Paulista de Odontologia 2002; 6:30-33.

Artigo apresentado em 16/10/2007

Aprovado em 24/09/2008

Versão final apresentada em 30/10/2010 